Bettina GRIMMER, Folgsamkeit herstellen. Eine Ethnographie der Arbeitsvermittlung im Jobcenter

\title{
Hadrien Clouet
}

\section{(2) OpenEdition \\ 1 Journals}

\section{Édition électronique}

URL : https://journals.openedition.org/ress/7743

DOI : $10.4000 /$ ress. 7743

ISBN : $1663-4446$

ISSN : $1663-4446$

Éditeur

Librairie Droz

Édition imprimée

Date de publication : 6 décembre 2021

Pagination : 328-331

ISSN : 0048-8046

Référence électronique

Hadrien Clouet, « Bettina GRIMMER, Folgsamkeit herstellen. Eine Ethnographie der Arbeitsvermittlung im Jobcenter ", Revue européenne des sciences sociales [En ligne], 59-2 | 2021, mis en ligne le 01 décembre 2021, consulté le 09 décembre 2021. URL : http://journals.openedition.org/ress/7743 ; DOI : https:// doi.org/10.4000/ress.7743

Ce document a été généré automatiquement le 9 décembre 2021.

(c) Librairie Droz 


\title{
Bettina GRIMMER, Folgsamkeit herstellen. Eine Ethnographie der Arbeitsvermittlung im Jobcenter
}

\author{
Hadrien Clouet
}

\section{RÉFÉRENCE}

Bettina GRIMMER, 2018, Folgsamkeit herstellen. Eine Ethnographie der Arbeitsvermittlung im Jobcenter, Bielefeld, Transcript, $281 \mathrm{p}$.

1 Depuis les années 2003-2005, une série de lois dites « Hartz » ont instauré un nouveau mot d'ordre dans le service public d'emploi allemand: "l'activation des chômeurs ", qui s'y décline sous le slogan "exiger et encourager » (fordern und fördern). Fruit d'un compromis entre l'approche libérale et social-démocrate, cette " activation » introduit des sanctions renforcées en échange d'une intervention accrue sur les personnes ellesmêmes, les forçant à devenir plus autonomes et responsables afin de compenser leurs défauts sur le marché de l'emploi. Elle repose essentiellement sur des convocations régulières obligatoires et individuelles des chômeurs, reçus par des conseillers personnalisés.

2 L'enquête sociologique menée par Bettina Grimmer prend le contre-pied du discours institutionnel de l'activation. Elle montre qu'il ne correspond en rien aux enjeux réels des interactions observables dans les Jobcenter, ces agences en charge des deux-tiers des chômeurs d'Allemagne - ceux qui ne sont pas éligibles à l'assurance-chômage, faute de durée suffisante de cotisation ou après expiration des droits. Pour elle, l'activation n'est pas une intervention donnant-donnant, mais un programme disciplinaire qui vise essentiellement l'inculcation d'une certaine docilité au public.

3 L'autrice s'appuie sur une enquête ethnographique dans des Jobcenter allemands, menée de manière symétrique auprès de chômeurs comme de conseillers. En 2008-2009, elle a accompli des entretiens dans plusieurs métropoles allemandes avec des responsables de 
partis, d'organisations caritatives et d'associations. Les deux années suivantes, elle a rejoint un groupe de bénévoles qui accompagnaient les chômeurs SGB II auprès des Jobcenter. Ce cadre lui a permis de conduire 18 observations participantes, enrichies de son propre matériau réflexif suite à des épisodes d'inscription épisodique en Jobcenter, complété par 20 entretiens complémentaires auprès de chômeurs. Elle enjambe par la suite le guichet pour suivre le quotidien de six conseillers à l'hiver 2011-2012.

Équipée de ce matériau, Bettina Grimmer livre une véritable sociologie de l'entretien et non de l'interaction. Si elle place l'entretien individuel entre conseiller et chômeur au centre de l'analyse, elle refuse de le rabattre intégralement sur l'ordre de l'interaction. Pour cela, elle organise l'ouvrage autour du triptyque «avant», " pendant » et « après » la rencontre bureaucratique, avant d'en dégager les conditions de réalisation ex-ante et les conditions de reproduction ex-post.

Avant un entretien, les individus (re)découvrent l'agence : la première partie y est consacrée. Dès que l'on entre dans un Jobcenter, l'organisation des locaux constitue un "script» (p. 38) limitant les interactions possibles. Le bâtiment est froid, le mobilier réduit à quelques chaises. Le public comprend que l'attractivité des lieux n'est pas une préoccupation et que l'attente représentera son activité principale. Il ne croisera guère les conseillers, qui empruntent des chemins séparés et ne discutent que derrière des portes closes - obtenir la clé des toilettes réservées aux conseillers constitue pour l'autrice, en début d'immersion, une des preuves de confiance les plus marquantes (p. 43). Dans ce monde triste, les bureaux des conseillers incarnent la seule touche de couleur et de vie. Mais pour y accéder, les usagers doivent s'inscrire à l'aide de multiples justificatifs. Ces derniers jouent trois rôles: sur le plan administratif, ils enregistrent le moindre contact pour le convertir en indicateur quantifié de performance; sur le plan économique, ils permettent immédiatement d'envoyer des offres d'emploi ; sur le plan symbolique, ils rappellent aux usagers le motif exclusif de leur présence, soit la recherche d'emploi. Mais les conseillers aussi s'activent en amont, en consultant les informations stockées sur les usagers. Au terme de ce double travail, d'inscription du côté des usagers et d'information du côté des conseillers, ceux-ci adressent un courrier de convocation à ceux-là - document intrigant, qui s'intitule « invitation »... mais rappelle en dernière page les sanctions encourues en cas d'absence. Tout est prêt pour un entretien, entre deux individus dotés de "savoirs asymétriques» (p. 79) : les chômeurs disposent d'un papier indiquant un lieu et une heure, mais ne connaissent ni le déroulé prévisionnel de l'entretien, ni leur interlocuteur. À l'inverse, armés d'archives informatiques tentaculaires, les conseillers ont analysé en détails leur profil.

6 La deuxième partie de l'ouvrage est dédiée au jour de la convocation, lorsque les chômeurs entrent dans le bureau et discutent avec les conseillers. Il ne s'agit pas encore d'un interrogatoire, mais déjà plus d'une discussion. L'enjeu apparaît simple à première vue, puisque les chômeurs s'efforcent de prouver leur volonté de reprendre un emploi aux conseillers. Mais un paradoxe pose problème: les conseillers doivent vérifier le comportement des chômeurs sur le marché de l'emploi, alors qu'ils ne les voient qu'au cours d'interactions administratives. Aussi doivent-ils exploiter l'entretien pour extrapoler des conclusions sur ce que font et ce que pensent les chômeurs loin d'eux, lorsqu'ils cherchent un emploi. En réalité, ils ne jugent donc jamais la volonté du public à travailler, mais uniquement la volonté du public à coopérer avec eux. 
7 Bettina Grimmer souligne ici les multiples incompatibilités de raisonnement entre conseillers et chômeurs. Certes, tous deux entendent aboutir à retrouver un emploi, mais avec une priorité différente : au plus vite pour les conseillers, de façon sécurisée pour les chômeurs. Les conseillers sont particulièrement exaspérés par les hésitations des chômeurs qui exercent un petit boulot et rechignent à le quitter pour une activité mieux rémunérée, par attachement à l'employeur et aux collègues, par facilité de transport ou par appréciation du geste professionnel pratiqué. L'activité de conseiller est donc essentiellement un «travail de persuasion» (p. 169). Seulement, les chômeurs ne sont pas toujours obéissants et lorsqu'ils résistent, toute la fiction du volontariat vole en éclats. Contrairement à ce qu'affirme la littérature sur la « coproduction de services", critiquée par l'autrice, il n'est nullement nécessaire que les deux interlocuteurs travaillent de concert : parfois, les conseillers adressent aux chômeurs des ordres qui ne laissent aucune place à la négociation. Pour cela, ils mettent en scène la supériorité du règlement sur leurs croyances personnelles et exposent, d'un ton neutre ou contrit, les retenues financières auxquelles s'exposent les usagers déviants. Ceux qui ne ploient pas s'exposent à des sanctions - sauf s'ils valident le diagnostic de leur conseiller pour mieux les convaincre qu'il peut être atteint par d'autres moyens.

8 Finalement, dans la troisième partie du livre, Bettina Grimmer aborde les suites de l'entretien. Le rendez-vous terminé, chacun part de son côté. Dans leur bureau, les conseillers continuent de réfléchir à l'entretien échu. Parfois, ils consultent à nouveau le profil des personnes les moins coopératives pour y chercher une explication. Certains d'entre eux défendent ici le recours aux sanctions, pour « les faire parler après l'expérience douloureuse de n'avoir pas assez d'argent »(p.189). Ils retranscrivent alors les grands axes de l'échange et sa conclusion, qui sera enregistrée dans le profil informatisé de l'usager, à l'instar de millions d'autres entretiens. Cela reproduit l'asymétrie initiale, puisque les chômeurs n'ont aucune idée des informations entreposées sur eux. Les chômeurs, quant à eux, quittent l'agence et rejouent l'entretien dans leur tête. Bettina Grimmer dégage trois ressentis possibles (nonexclusifs). L'entretien peut apparaître comme un rituel de contrôle, consistant à présenter des justificatifs à un conseiller impuissant ( «je n'ai rien pour vous, vous pouvez rentrer, au revoir », p. 203). L'entretien peut aussi constituer un moment rassurant, où les chômeurs peuvent confier leurs problèmes à des conseillers compréhensifs, voire prêts à transgresser les règles pour aider les usagers (« elle est super cool et me comprend», p. 213). Finalement, l'entretien condense une expression de pouvoir, au sens wébérien du terme, où un conseiller impose à un chômeur d'agir d'une certaine manière, ce qui entraîne de vives répercussions émotionnelles (« c'est très dur d'y aller, car j'ai très peur ", p. 218).

9 Au terme de cette description fine, Bettina Grimmer souligne que contrairement au discours institutionnel, les entretiens n' " activent " pas les personnes, dans le sens où elles leur donneraient l'énergie ou la capacité d'agir. Ils leur inculquent plutôt la conformation et la soumission aux règles de l'institution. Le bon chômeur est un individu fiable, courtois, qui gère ses affaires de façon ordonné, envoie des informations par anticipation et surjoue le respect des règles. La répétition des entretiens en face-à-face accrédite l'idée d'une cause individualisée du chômage, en proposant comme unique horizon aux populations accueillies de "résorber leurs déficits personnels » (p. 243). 
10 Cette sociologie de la docilité n'est toutefois pas qu'une sociologie de la domination. En effet, Bettina Grimmer montre que la docilité est un objectif partagé. Pour les conseillers, elle indique que le chômeur est un "bon client ", soucieux de retrouver un emploi et de suivre ses recommandations, qui ont donc un sens et ne constituent pas des paroles en l'air. Pour les chômeurs, elle atténue la contrainte exercée par les conseillers et suscite leur confiance, voire leur estime.

11 Si l'autrice expose comment les chômeurs et les conseillers gèrent la conditionnalité des aides, elle souligne surtout que les devoirs des premiers dépendent largement de la personne qui leur fait face. Cela dit, on pourra ici regretter que la piste reste ébauchée, puisque l'autrice n'explique pas quelles dispositions personnelles se traduisent en logiques d'action face aux chômeurs, et comment - effets de génération, de socialisation primaire, de rapport à l'écrit, de capital culturel ? L'ordre de l'interaction prime ici (trop) largement sur l'ordre social et l'ordre institutionnel. D'où viennent les individus et de quoi rêvent-ils en-dehors des interactions? Cela aurait aussi permis de dégager les effets proprement marchands de telles interactions, dont on saisit bien l'aspect disciplinaire répété systématiquement, sans forcément comprendre leur conséquence en matière de dégradation de l'emploi. Concentrée sur le matériau interactionnel, cette sociologie des Jobcenter ne fournit pas d'économie politique de l'intermédiation.

12 Ces pistes de discussion n'enlèvent rien à la contribution décisive de l'autrice pour prendre le contre-pied de la littérature qui réduit les lois Hartz allemandes à une dimension exclusivement "néolibérale ». Bien sûr, la rhétorique initiale au fondement des Jobcenter prône la flexibilité et la responsabilité individuelle. Mais son projet est inachevé : aucun chômeur n'est transformé en entrepreneur avide de risque, prêt à tout dans l'espoir d'un gain ultérieur. Pour les conseillers, il s'agit plutôt de conformer les chômeurs à l'idéal de l'employé docile et discipliné, ordonné et digne de confiance. Cette promotion de l'obéissance est aussi leur manière - souvent ambigüe, parfois violente - de protéger les chômeurs du projet néolibéral.

\section{AUTEURS}

\section{HADRIEN CLOUET}

Université de Toulouse Jean Jaurès - CERTOP 